\title{
Open clusters: their kinematics and metallicities
}

\author{
L. Chen ${ }^{1}$, J. L. Hou ${ }^{1}$, J. L. Zhao ${ }^{1}$ and R. de Grijs ${ }^{2}$ \\ ${ }^{1}$ Shanghai Astronomical Observatory, Chinese Academy of Sciences, 80 Nandan Road, \\ Shanghai 200030, China \\ email: chenli@shao.ac.cn, houjl@shao.ac.cn, jlzhao@shao.ac.cn \\ ${ }^{2}$ Department of Physics and Astronomy, University of Sheffield, Hicks Building, Hounsfield \\ Road, Sheffield S3 7RH, UK; and National Astronomical Observatories, Chinese Academy of \\ Sciences, 20A Datun Road, Chaoyang District, Beijing 100012, China \\ email: r.degrijs@sheffield.ac.uk
}

\begin{abstract}
We review our work on Galactic open clusters in recent years, and introduce our proposed large program for the LOCS (LAMOST Open Cluster Survey). First, based on the most complete open clusters sample with metallicity, age and distance data as well as kinematic information, some preliminary statistical analysis regarding the spatial and metallicity distributions is presented. In particular, a radial abundance gradient of $-0.058 \pm 0.006 \mathrm{dex} \mathrm{\textrm {kc } ^ { - 1 }}$ is derived. By dividing clusters into the age groups we show that the disk abundance gradient was steeper in the past. Secondly, proper motions, membership probabilities, and velocity dispersions of stars in the regions of two very young open clusters are derived. Both clusters show clear evidence of mass segregation, which provides support for the "primordial" mass segregation scenario. Based on the advantages of the forthcoming LAMOST facility, we have proposed a detailed open cluster survey with LAMOST (the LOCS). The aim, feasibility, and the present development of the LOCS are briefly summarized.
\end{abstract}

Keywords. Galaxy: disk, open clusters and associations: general, open clusters and associations: individual (NGC2244), open clusters and associations: individual (NGC6530)

\section{The open cluster system and the observational database}

Open clusters (OCs) are considered excellent laboratories for studies of stellar evolution. Studies in the research area dealing with OCs show a rapid growth in 1990's and this area continues to develop vigorously. There may be several reasons for this recent growth of OC studies. New techniques are greatly beneficial to the OC observations, including, for example, the application of wide-field, high quality CCD cameras and, more recently, multi-object spectrographs. In addition, OC studies play a very important and unique role in determining the structure and evolution of the Galactic disk.

OCs have long been used to trace the structure and evolution of the Galactic disk. From the observational point of view, there are some important advantages of using OCs as opposed to field stars. In OCs, we deal with groups of stars of nearly the same age, a similar composition and at a similar distance. We can observe OCs to large distances, and the photometric distances to most of the OC sample have already been derived. In particular, OCs have a relatively stable orbital motion, which can be used as a better tracer of the Galactic disk structure. OCs also have a wide range of ages, so that - combined with their spatial distribution and kinematic information - we can study the effects of dynamical evolution. Very young OCs are ideal objects for studies of the stellar initial mass function. Furthermore, when combined with abundance data, we can 


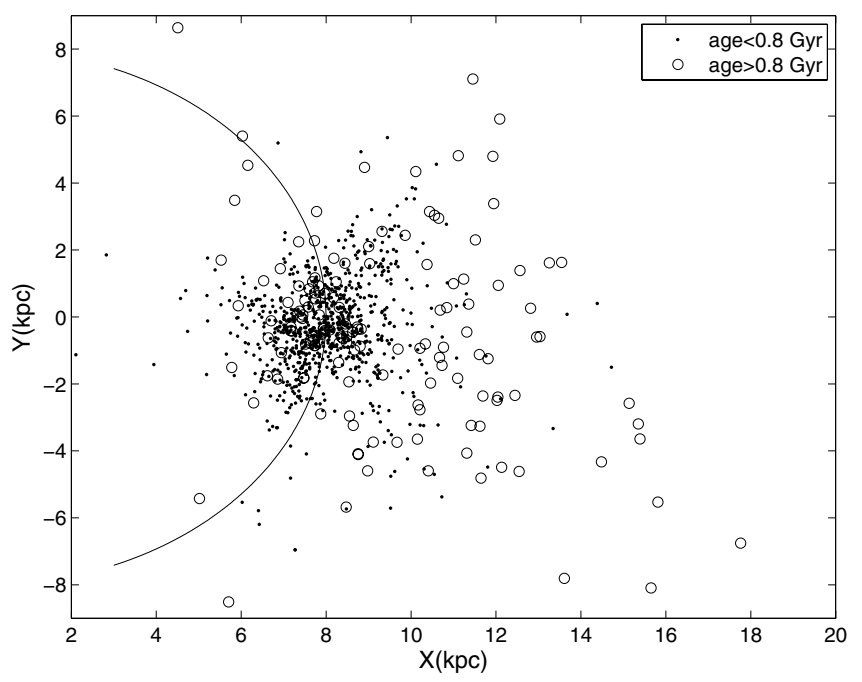

Figure 1. Spatial distribution of OCs in the Galactic $(X, Y)$ plane. The open circles and dots are the older and younger clusters, respectively. The large semi-circle has a radius of $8.0 \mathrm{kpc}$, centered on the Galactic center.

investigate the chemical evolution history of the Galactic disk (Carraro et al. 1998, Chen et al. 2003).

At present, the total number of detected clusters and associations is around 1700 (Dias et al. 2002), about $60 \%$ of which have distance and age information and for about half of them have proper motions available. Less than one-fourth of the sample have both proper motion and radial velocity parameters, and only a small portion (about 8\%) of the OCs have well-determined mean metallicities. We have compiled an updated OC catalog (Chen et al., in prep.), for which data have been extracted from various sources mainly from (Dias et al. 2002), (Kharchenko et al. (2005a), Kharchenko et al. (2005b)), and the WEBDA database (Mermilliod \& Paunzen 2003). Based on this large sample, we can embark on statistical studies of the Galactic OC system.

\section{Spatial and kinematic properties of Galactic Open clusters}

Using our updated database for 993 OCs with distance and age data, we plotted the cluster positions on an $(X, Y)$ coordinate system, with a zero point at the Galactic Center (where $R_{\odot}=8.0 \mathrm{kpc}$ ), as shown in Fig. 1. The arc in Fig.1 represents the solar circle about the Galactic Center. One can see from this figure that there are very few OCs at the galactocentric distances less than $5 \mathrm{kpc}$. Furthermore, while young clusters (with ages less than 0.8 Gyr; see Phelps et al. 1994) are distributed quite uniformly around the Sun, most of the older OCs are distributed in the outer part of the disk. Such apparent distributions are partially due to the much higher extinction in the direction of Galactic Center and the deficiency of older clusters in the inner part of the disk, that has been attributed to the preferential destruction of these clusters by giant molecular clouds, which are primarily found in the inner Galaxy.

Regarding the spatial distribution perpendicular to the Galactic plane, most OCs represent the typical thin-disk population, with a scale height of about $66 \mathrm{pc}$. However, the subsample of old OCs, most of which are found in the outer disk, has a much larger scale height of $221 \mathrm{pc}$. These scale heights are in excellent agreement with the earlier results of Janes et al. (1988) and Janes \& Phelps (1994). 

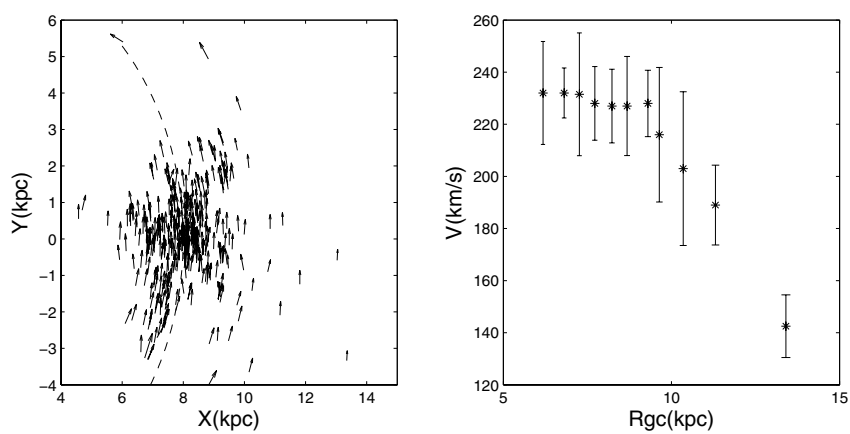

Figure 2. Left: Velocity projection on the Galactic plane of 369 clusters. The Sun is located at $(X=8, Y=0) \mathrm{kpc}$. Right: Rotation velocity of the OCs as a function of galactocentric distance

In our kinematic sample, we have compiled a total of 369 OCs for which a distance and both proper motion and radial velocity data are available. In left-hand panel of Fig. 2 , we plot their projected velocity onto the disk, whilst the right-hand panel shows the averaged rotation velocity of OCs as a function of the galactocentric distance.

Based on our kinematic dataset we can also constrain the Galactic differential rotation, as well as the Galactic radial motion parameters in the solar neighborhood. We have compiled a subsample of 117 clusters with heliocentric distances of $0.5-2.0 \mathrm{kpc}$ and ages younger than $0.8 \mathrm{Gyr}$, which can be considered as typical thin-disk objects in the Galaxy, for which the Oort's theory is applicable. The kinematical parameters determined from these clusters can be used to represent the kinematic properties of thin-disk objects in the vicinity of the Sun. Thus, we deduced the following Galactic parameters: (i) the mean heliocentric velocity of the OC system, $(u 1, u 2, u 3)=(-16.1 \pm 1.0,-7.9 \pm 0.4,-10.4 \pm$ $1.5) \mathrm{km} \mathrm{s}^{-1}$, (ii) the characteristic velocity dispersions, $\left(\sigma_{1}, \sigma_{2}, \sigma_{3}\right)=(17.0 \pm 0.7,12.2 \pm$ $0.9,8.0 \pm 1.3) \mathrm{km} \mathrm{s}^{-1}$, (iii) the Oort constants, $(A, B)=(14.8 \pm 1.0,-13.0 \pm 2.7) \mathrm{km} \mathrm{s}^{-1}$ $\mathrm{kpc}^{-1}$, and (iv) the radial motion parameters, $(C, D)=(1.5 \pm 0.7,-1.2 \pm 1.5) \mathrm{km} \mathrm{s}^{-1}$ $\mathrm{kpc}^{-1}$ (Zhao et al. 2006). The parameters determined from these clusters have accuracies significantly better than those obtained from the other groups of clusters.

\section{The disk abundance gradient based on open clusters}

OCs can also be used as a powerful tool to understand whether and how the spatial abundance distribution changes with time, because OCs have formed at all epochs and since their ages, distances, and metallicities can be derived more reliably than the equivalent parameters of field stars.

We have compiled a full OC sample, containing 144 objects, with metallicity, distance and age parameters. From this sample, we obtain a radial metallicity gradient of $-0.058 \pm$ 0.006 dex kpc $\mathrm{k}^{-1}$ (Chen et al., in prep.), for galactocentric distances ranging from about $7 \mathrm{kpc}$ to $17 \mathrm{kpc}$. By dividing the clusters into young and old subsamples (e.g., Chen et al. 2003), we find that the corresponding gradients are significantly different, as shown in the upper panel of Fig. 3. That is, the gradient is steeper in the past, and shallower for younger clusters. In the bottom panel of Fig. 3, the gradients of the inner and outer subsamples have similar values.

These abundance gradient results are consistent with those from the HII regions (Deharveng et al. 2000) or planetary nebula data (Maciel et al. 2006).

In a recent review, Maciel et al. (2006) combined abundance data from planetary nebula and $\mathrm{OC}$ samples to provide observational constraints on disk chemical evolution models. 


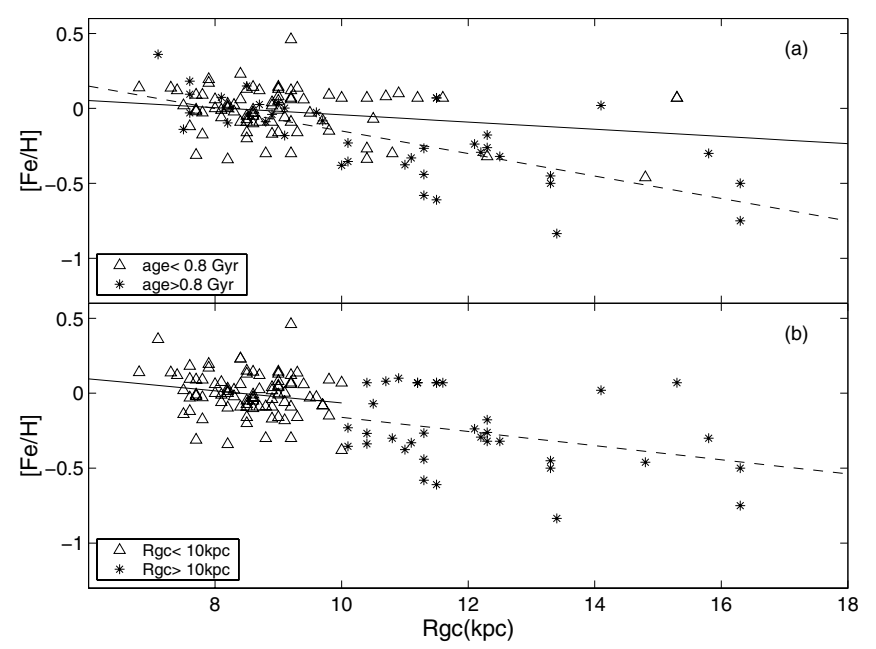

Figure 3. (a) Time evolution of the $[\mathrm{Fe} / \mathrm{H}]$ gradient. Triangles and stars represent younger and older OCs, respectively. (b) Gradients for inner-disk and outer disk clusters. (Chen et al. 2003)

As shown in Fig. 10 of their paper, these authors investigated the abundance gradient as a function of time by combining the results derived from OC data by Friel et al. (2002), and by Chen et al. (2003). The theoretical tracks of Chappini et al. (2001) and Hou et al. (2000), are also shown, respectively. The differences between these two model predictions are rather large. In the model by Hou et al. (2000), based on an exponentially decreasing infall rate and an "inside-out" scenario for the formation of the Galactic disk, a rapid increase of metal abundances at early times in the inner disk caused a steep gradient. As the star formation activity migrated to the outer disk, the abundances got enhanced in that region as well, so that the gradients flatten out. In the model used by Chiappini et al. (2001), two infall episodes are assumed to form halo and the disk. The disk is also formed in an "inside-out" formation scenario, in which the time-scale is a linear function of the galactocentric distance. In general, some steepening of the gradient is predicted by that model. It seems that the Hou et al. (2000) model is better supported by the observational data.

Thus, based on OC data we may constrain the disk chemical evolution such that in the early stages of disk formation it showed a steeper abundance gradient, while later on the gradient became flatter. These inferences are not very conclusive, since we still do not have a sufficiently large sample of outer disk clusters. The compilation of such a sample is critical for these statements to be made firmer, and we propose to focus on obtaining such a sample as a priority in this field.

As regards the overall gradient along the disk, Twarog et al. (1997) proposed a step function for the disk abundance. That is, inside $10 \mathrm{kpc}$, there is a shallow gradient, while beyond $10 \mathrm{kpc}$ the sample is too small to determine, if a gradient exists at all. They used a sample of 14 OCs in the outer disk. However, considering that we now have 34 OCs beyond $10 \mathrm{kpc}$, our conclusion of a continuous linear gradient is more likely.

\section{Very young open clusters and the effects of mass segregation}

In some previous work on young open clusters, including the Orion Nebula Cluster (Hillenbrand 1997) and R136 (e.g. Cambell et al.1992, Brandl et al. 1996) the authors found evidence of mass segregation. However, the question is, for such young star clusters, 


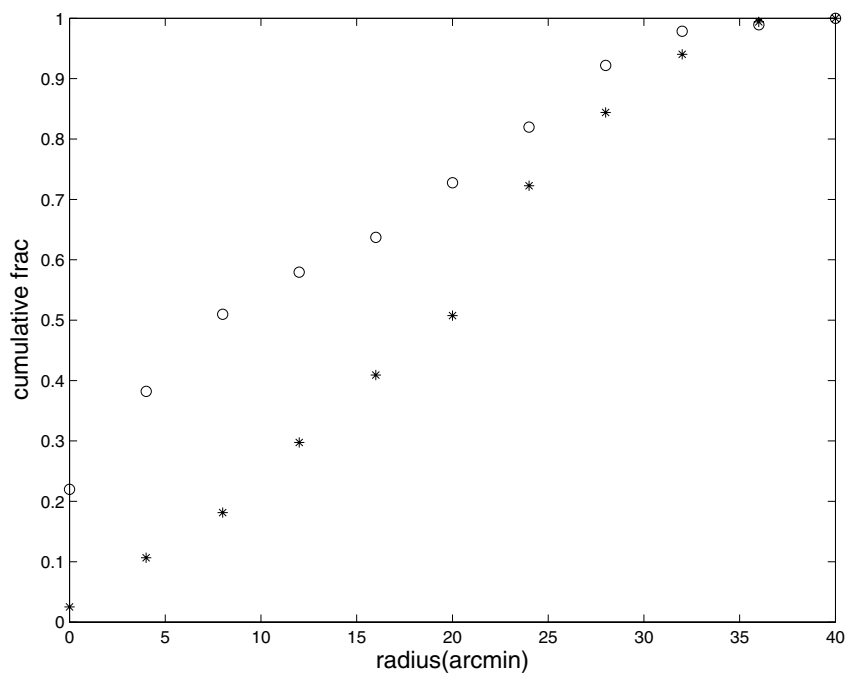

Figure 4. Normalized cumulative radial number density profile for NGC 6530 members with $m_{B} \leqslant 12(\mathrm{o})$ and $m_{B}>12(*)$. Result from Chen et al. (2007)

whether these effects are mainly the result of dynamical evolution or possibly primordial in nature.

Recently, we investigated two very young open clusters, NGC 2244 and NGC 6530, both with ages younger than $5 \mathrm{Myr}$. As an example, here we show some results for NGC 6530 (Chen et al. 2007).

The observational data were taken from our historical photographic plates at Shanghai Observatory, with a time baseline of 87 years. The old photographic plates were taken by the 40 -cm refractor at Sheshan observational station.

From these photographic data, we determined proper motions for 364 stars in a one square degree region centered on the cluster, and estimated their membership probabilities. Since these cluster stars are located at the same distance, and because most of them are main sequence stars, we can use luminosity instead of mass to study the (mass/luminosity) segregation effects. Figure 4 shows the cumulative radial number density profile for bright and faint cluster stars, which shows that brighter (or massive) stars are more concentrated towards the inner part of the cluster. this indicates an evident luminosity or mass segregation effect. A similar result was obtained for our other sample cluster, NGC 2244 (Chen et al. 2007).

We conclude that both OCs, NGC 6530 and NGC 2244, show clear evidence of mass (luminosity) segregation. These two clusters are too young for them to have been shaped by dynamical relaxation processes. The observed segregation might be due to the combination of both initial conditions and dynamical evolution. Therefore, our results support the "primordial mass segregation" scenario, such as the "competitive accretion" model. (see Larson 1991, Bonnell et al. 2001a, Bonnell et al. 2001b)

\section{The LOCS - LAMOST Open Cluster Survey project}

Regarding the present status of our OC database, we need a much larger sample with both abundance and three-dimensional spatial motion data in order to study the global disk dynamical and chemical evolution. In particular, to study the disk chemical evolution, we need more outer-disk cluster data so that we can determine the abundance 
gradient evolution decisively. Equally important, uniform observational data sets for different clusters are very much needed. As for a single cluster, in many cases only a few stars are observed spectroscopically to derive the average metallicity and mean radial velocity. On the whole, a large and homogenous spectroscopic data set for OCs is non-existent yet.

To improve the situation, there are some important OC projects ongoing or in the planning phases. These includes, for example, WOCS (The WIYN Open Cluster Study; Mathieu 2000), which aims to obtain comprehensive and definitive astrometric, photometric and spectroscopic databases for several fundamental clusters; SOCS (SEGUE Open Cluster Survey), which, as part of the AS-2 project, is designed to obtain radial velocities and metallicities for more than 100 OCs. The Italian BOCCE project (Bragaglia 2006), by obtaining photometric and high-resolution spectroscopic data of 30 clusters, is performing a detailed investigation of their ages, distances, and abundances.

Recently we proposed the LOCS project - the LAMOST Open Cluster Survey. The Large Sky Area Multi-Object Fiber Spectroscopic Telescope (LAMOST) project (see http://www.lamost.org/en/) is one of the National Major Scientific Projects undertaken by the Chinese Academy of Science. LAMOST is a 4 -meter aperture quasi-meridian reflecting Schmidt telescope, with a large field of view of about 20 square degree, and 4000 fibers.

The telescope is located at the Xinglong Observing Station of the National Astronomical Observatories. At present, a small system with an effective 2-meter aperture instrument has already obtained first light. The full system is scheduled to be finished in early 2009.

For the LOCS, the main advantage of using LAMOST is that it can be most efficient for an OC survey. With its multiple fibers and large field of view, we can complete spectroscopic survey observation for at least one cluster per observing night. This type of survey observations will have deep enough magnitude limits to reach most of the old clusters within $5-8 \mathrm{kpc}$.

We expect the LOCS, in about 4-years of observations, to survey a total of amount six hundred OCs, each covering one square degree of the sky. We plan to obtain homogeneous metallicities and radial velocities of stars in each cluster. This will lead to reliable membership determinations, which will, in turn, form a fundamental basis for OC studies. Obtaining many more abundance and kinematic properties of old and distant OCs provides crucial constraints for modelling of the structure and evolution of the Galaxy.

From the selected OC targets, we choose 150 OCs with suitable ages and distances as the first-priority sample for LOCS observations. Another 13 well-studied OCs have been chosen as standard objects for comparison. We have already prepared input catalogs for stars in the selected clusters, and a preliminary membership estimation for candidate clusters is being derived.

\section{Acknowledgements}

LC, JLH and JLZ are supported by the NSFC (Grant Nos. 10773021, 10573028,10333050, 10333020,10573022) and NKBRSFG 2007CB815402 and 403. RdG was partially supported by an "International Joint Project" grant, jointly funded by the Royal Society in the UK and the Chinese National Science Foundation (NSFC). This research has made use of the WEBDA database, operated at the Institute for Astronomy of the University of Vienna. 


\section{References}

Bonnell, I. A., Bate, M. R.,Clarke, C. J., \& Pringle, J. E. 2001a, MNRAS, 323, 785

Bonnell, I. A., Clarke, C. J., Bate, M. R., \& Pringle,J. E. 2001b, MNRAS, 324, 573

Brandl, B., Sams, B. J., Bertoldi, F., Eckart, A.,Genzel, R., Drapatz, S., Hofmann, R., Löwe, M., \& Quirrenbach, A. 1996, ApJ, 466, 254

Bragaglia, A. \& Tosi, Monica. 2006, AJ, 131, 1544

Campbell, B., et al. 1992, AJ, 104, 1721

Carraro, G., Ng, Y. K., \& Portinari, L. 1998, MNRAS,296, 1045

Chen, L., de Grijs, R., \& Zhao, J. L. 2007, AJ, 134, 1368

Chen, L., Hou, J. L., \& Wang, J. J. 2003, AJ, 125, 1397

Chiappini, C., Matteucci, F., \& Romano, D. 2001, ApJ, 550, 1044

Deharveng, L., Peña, M., Caplan, J., Costero, R. 2000, MNRAS, 311, 329

Dias, W. S., Alessi, B. S., Moitinho, A., \& Lépine, J. R. 2002, A\& A, 389, 871

Friel, E. D., Janes, K. A., Tavarez, M., et al. 2002, AJ, 124, 2693

Hillenbrand, L. A. 1997, AJ, 113, 1733

Hou, J. L., Prantzos, N., \& Boissier, S. 2000, A\&A, 362, 921

Janes, K. A., Tilley, C., \& Lyngå, G.1988, AJ, 95, 771

Janes, K. A. \& Phelps, R. L. 1994, AJ, 108, 1773

Kharchenko, N. V., Piskunov, A. E., Roeser, S., Schilbach, E., \& Scholz, R.-D. 2005, A $\& A$ A 438, 1163

Kharchenko, N. V., Piskunov, A. E., Roeser, S., Schilbach, E., \& Scholz, R.-D. 2005, A\&SA, 440, 403

Larson, R. B. 1991, in: Falgarone, E., Boulanger, F., \& Duvert, G., (eds.), Fragmentation of Molecular Clouds and Star Formation, IAU Symp. 147 (Dordrecht: Kluwer), p. 261

Maciel, W. J., Lago, L. G., \& Costa, R. D. D. 2006, A\&SA, 453, 587

Mathieu, R. D. 2000, in: R. Pallavicini, G. Micela, \& S. Sciortino, (eds.), Stellar Clusters and Associations: Convection, Rotations, and Dynamos, ASP Conference Series, p. 517

Mermilliod, J.-C. \& Paunzen, E. 2003, A\& A, 410, 511

Phelps, R. L., Janes, K. A., \& Montgomery, K. A. 1994, AJ, 107, 1079

Twarog, B. A., Ashman, K. A., \& Anthony-Twarog, B. J. 1997, AJ, 114, 2556

Zhao, J. L., Chen, L., \& Zu, Z. L. 2006, Chin. J.Astron.Astrophysics, 6, 287 